\title{
Characteristic imaging features of clonorchiasis
}

\author{
WK Lo *, SM Yu, James CS Chan
}

\author{
Hong Kong Med J 2018;24:206.e3-4 \\ DOI: $10.12809 / \mathrm{hkmj} 166198$
}

\begin{abstract}
A 43-year-old Chinese man presented to United Christian Hospital, Hong Kong, in January 2016, with a 2-year history of vague right upper quadrant pain. Blood test results were unremarkable except for an episode of transient eosinophilia. Ultrasonography showed mildly dilated intrahepatic ducts. Magnetic resonance cholangiopancreatography showed diffuse and uniform mild dilatation of periphera intrahepatic bile ducts (Fig 1) consistent with the 'too many intrahepatic ducts' sign (Fig 2). Notably, there was no central extrahepatic bile duct dilatation. Subsequently requested stool microscopy was positive for Clonorchis sinensis ova. The patient was given a course of praziquantel.

Clonorchiasis is a foodborne zoonosis caused by consumption of raw or undercooked freshwater
\end{abstract}

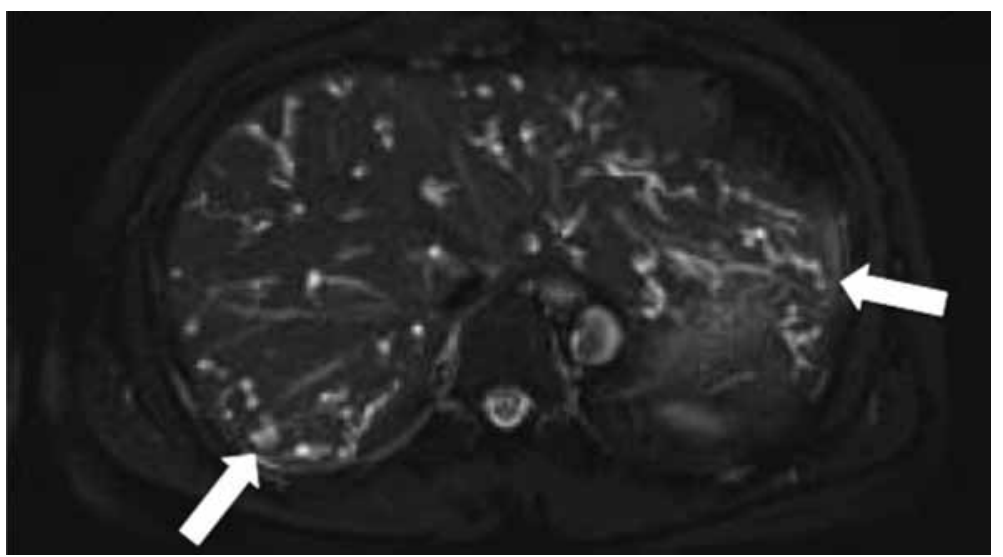

FIG I. Axial T2-weighted magnetic resonance image showing dilatation of the peripheral intrahepatic ducts (arrows)

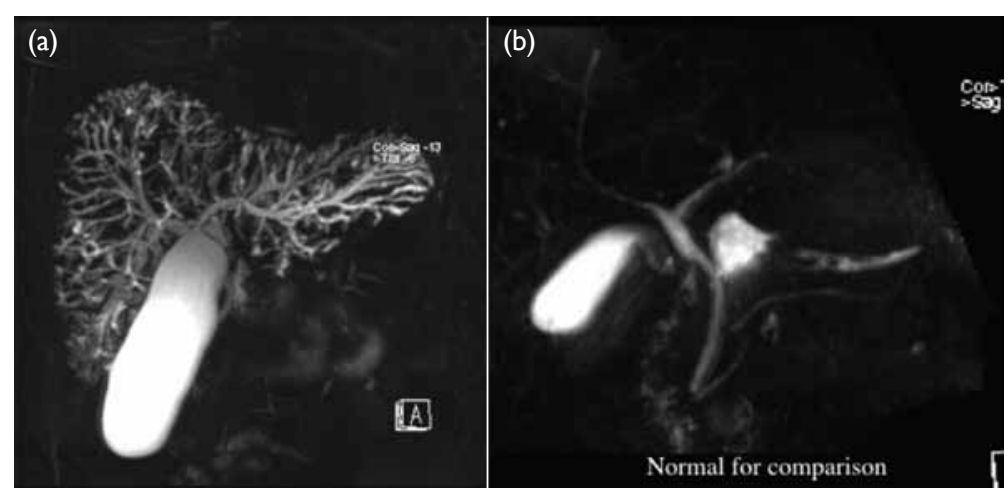

FIG 2. Reformatted magnetic resonance cholangiographic images showing the 'too many intrahepatic ducts' sign in a patient with clonorchiasis (a), compared with a healthy individual (b) fish infested by the liver fluke $C$ sinensis. $C$ sinensis larvae penetrate the scales of freshwater fish and encyst in subcutaneous tissues. Cysts, when consumed by a definitive host, hatch in the intestine and migrate to the biliary tree, where the adult flukes establish residence. Clonorchiasis is endemic in many parts of Asia, including China, Korea, and Vietnam. ${ }^{1}$ Clonorchiasis and its complications are not commonly seen in affluent populations, such as those in Hong Kong. However, the increasing mobility of people around the region means that clinicians should be vigilant of the condition and alert to its symptoms. Knowledge of the characteristic imaging features of clonorchiasis would prompt stool microscopy and allow early diagnosis.

Within the biliary tree, adult $C$ sinensis flukes reside in small- or medium-sized peripheral intrahepatic ducts. The organisms cause dilatation of the intrahepatic ducts. In heavy infestations, the extrahepatic ducts, the gallbladder, or even the pancreatic duct can also be involved. The dilated peripheral ducts can be visualised by direct cholangiography, or to a better extent by magnetic resonance cholangiopancreatography (owing to the independence of rate and volume of the contrast injection), as the 'too many intrahepatic ducts' sign. ${ }^{2}$ Occasionally, linear or elliptical filling defects representing the flukes can be seen. ${ }^{3}$

Light infestations with $C$ sinensis can be asymptomatic. Heavier infestations can result in fever, jaundice, right upper quadrant pain, and eosinophilia. Untreated, chronic clonorchiasis induces chronic inflammation of the bile ducts. Recurrent pyogenic cholangitis, cholelithiasis, pancreatitis, and cholangiocarcinoma are the main complications. ${ }^{4}$

Infestations are diagnosed by observation of $C$ sinensis ova during stool microscopy. Immunological and molecular techniques of diagnosis are still at the experimental stage. ${ }^{1}$ Praziquantel is the only drug treatment for clonorchiasis that is recommended by the World Health Organization. ${ }^{1}$

In summary, clinicians in Asia should be vigilant of clonorchiasis. In modern clinical practice, magnetic resonance cholangiopancreatography is often requested for non-invasive evaluation of the biliary tree. Knowledge and recognition of the characteristic imaging features of clonorchiasis would prompt timely investigation by stool microscopy. Early diagnosis and treatment of clonorchiasis can prevent complications such as recurrent pyogenic cholangitis and cholangiocarcinoma. 


\section{Declaration}

The authors have no conflicts of interest to disclose.

${ }^{1}$ WK Lo *, MB, BS, FRCR

${ }^{2}$ SM Yu, FRCR, FHKAM (Radiology)

2 JCS Chan, FRCR, FHKAM (Radiology)

${ }^{1}$ Department of Diagnostic and Interventional Radiology, Kwong Wah Hospital, Yaumatei, Hong Kong

${ }^{2}$ Department of Radiology and Organ Imaging, United Christian Hospital, Kwun Tong, Hong Kong

* Corresponding author: waikglo@gmail.com

\section{References}

1. World Health Organization. Foodborne trematode infections-clonorchiasis. Available from: http://www. who.int/foodborne_trematode_infections/clonorchiasis/ en/. Accessed on 27 Nov 2016.

2. Choi D, Hong ST. Imaging diagnosis of clonorchiasis. Korean J Parasitol 2007;45:77.

3. Lim JH. Radiologic findings of clonorchiasis. AJR Am J Roentgenol 1990;155:1001-8.

4. Choi BI, Han JK, Hong ST, Lee KH. Clonorchiasis and cholangiocarcinoma: etiologic relationship and imaging diagnosis. Clin Microbiol Rev 2004;17:540-52. 